\title{
Exact Solutions of Compressible Flow Equations with Spherical Symmetry
}

\author{
By P. L. Sachdev, K. T. Joseph, and M. Ejanul Haque
}

In this paper, we construct spherically symmetric solutions of the equations of compressible flow, which are important in the theory of explosion waves in air, water, and other media. Following McVittie [1], we write a general solution form, in terms of velocity potential, as a product of a function of time and a function of a similarity variable. First, we find solutions to the equations of motion and continuity without reference to adiabatic or isentropic relation. These solutions are quite general and can be applied to nonadiabatic motions, such as the motions of interstellar gas clouds that lose energy by radiation. All the solutions found by McVittie [1] have linear velocity profile with respect to distance. We introduce a nonlinear form of the velocity function containing an arbitrary function of the similarity variable. Adiabatic conditions lead to a second-order ODE, which we discuss in some detail. We relate our work to the earlier investigations of Taylor [2], McVittie [1], and Keller [3].

\section{Introduction}

The system of nonlinear partial differential equations describing gas flows in spherical symmetry is important for the theory of explosion in air, water, and other media and has been studied for a long time (Courant and Friedrichs [4]

\footnotetext{
Address for correspondence: P. L. Sachdev, Department of Mathematics, Indian Institute of Science, Bangalore 560012, India; e-mail: sachdev@math.iisc.ernet.in 
and Stanyukovich [5]). Such motions can be considered analogous to onedimensional motions in a tube under the influence of a piston. In the three-dimensional case, the piston is replaced by an expanding or contracting sphere, which impresses motion on the medium inside or outside. Some of the earlier analytic studies used self-similarity to reduce the problem to a system of nonlinear ordinary differential equations subject to boundary conditions at the shock and at the piston (Sedov [6], Taylor [2], Keller [3], and McVitte [1]). In this paper, we attempt to find more general exact solutions of gas dynamics equations in spherical symmetry. In the present context, we refer in particular to the work of McVittie [1] and Keller [3]. McVittie [1] gave an explicit form of solution for the three-dimensional case for equations of continuity and motion but restricted his analysis to the spherically symmetric case only. We generalize the work of McVittie [1] and Keller [3] to find exact solutions of compressible gas dynamics equations in spherical geometry. Here, $p$ is the pressure, $\rho$ the density, and $q$ the particle velocity. $r$ is the radial distance measured from the center of the sphere; $t$ stands for time. The equations of continuity and motion are

$$
\partial_{t} \rho+\frac{1}{r^{2}} \partial_{r}\left(r^{2} \rho p\right)=0
$$

and

$$
\partial_{t} q+q \partial_{r} q=-\frac{1}{\rho} \partial_{r} p
$$

respectively. If $S$ is the entropy per unit mass of gas and $\gamma$, the ratio of the specific heats, then the rate of entropy per unit mass of the gas is given by

$$
\frac{d S}{d t}=\frac{d}{d t}(\log p-\gamma \log \rho)
$$

and, therefore, for the adiabatic motion, we have

$$
\frac{d S}{d t}=\frac{d}{d t}(\log p-\gamma \log \rho)=0 .
$$

Motivated by the "Einstein's gravitational potential function," McVittie [1] showed that, for spherical symmetry,

$$
q=-\phi_{r t} / \nabla^{2} \phi, \quad \rho=-\nabla^{2} \phi, \quad p=P-\phi_{t t}+2 \int \frac{I}{r} d r+I,
$$

expressed in terms of an arbitrary function $\phi(r, t)$ satisfy the PDEs (1) and (2), where

$$
I=\left(\phi_{r t}\right)^{2} / \nabla^{2} \phi, \quad \nabla^{2} \phi=\left(\frac{\partial}{\partial r}+\frac{2}{r} \frac{\partial}{\partial r}\right) \phi .
$$

McVittie [1] assumed the potential function $\phi$ to be a product of a function of time and a function of a similarity variable. 
He considered two special cases: (i) the function of similarity variable is known but the function of time is unknown, (ii) the function of the similarity variable is arbitrary but the function of time is known. Then, he obtained solutions for both cases. These solutions have a linear distribution of particle velocity. McVittie [1] imposed the particle adiabatic condition on both the cases (i) and (ii) and obtained some special explicit solutions. He then applied them to flows with shocks and vacuum fronts. Although the basic idea of expressing the solution of the equations of motion and continuity in terms of a potential function is ingenious, there is a need to generalize the work of McVittie [1]. All the solutions found by him have a linear velocity profile with respect to distance. These solutions must be generalized to be applicable to more realistic situations. In the present study, we extend the work of McVittie [1] and Keller [3], and construct solutions for which the velocity function is nonlinear in distance.

\section{Exact solutions in spherical symmetry}

We begin with the mathematical formulation and summary of the work of McVittie [1]. He developed a method to find a very general form of the solution of (1) and (2) in terms of a velocity potential $\phi$; he also assumed that $\phi(r, t)=$ $f(t) w\left(r t^{-\alpha}\right)$. McVittie [1] then proved the following result.

With $x=r t^{-\alpha}, f$ a function of $t$ alone, and $w$ a function of $x$, McVittie [1] showed that the velocity $q$, density $\rho$, and pressure $p$ given by

$$
\begin{gathered}
q=\alpha t^{\alpha-1} x^{g+2} \frac{\left(x^{(1-g)} w_{x}\right)_{x}}{\left(x^{2} w_{x}\right)_{x}} \\
\rho=f t^{-2 \alpha} x^{-2}\left(x^{2} w_{x}\right)_{x} \\
p=p_{0}+\alpha^{2} f t^{-2}\left[\frac{\left(\alpha g^{2}-g+t g_{t}\right)}{\alpha)} w-\left(2 g-\frac{1}{\alpha}\right) x w_{x}\right. \\
\left.+x\left(x w_{x}\right)_{x}-x^{g+2} u-2 \int^{x} x^{g+1} u d s\right]
\end{gathered}
$$

satisfy (1) and (2), where $\alpha$ is a constant and the functions $w, u, f$, and $g$ are related by

$$
\begin{aligned}
& g=t \frac{f_{t}}{\alpha f} \\
& u=\frac{v_{x}^{2}}{x v_{x}+(g+1) v} \\
& v=x^{1-g} w_{x}
\end{aligned}
$$


McVittie [1] found special solutions of the form (6)-(8) for which $q$ is linear in $r$; these solutions satisfy the adiabatic condition (3):

$$
\left(\partial_{t}+q \partial_{r}\right)(\log p-\gamma \log \rho)=0 .
$$

More precisely, he found the following classes of solutions.

Case A:

$$
\begin{aligned}
& q=\frac{a(t) r}{t} \\
& \rho=(\lambda+1) f(t) t^{2 \alpha} x^{\lambda-2}, \\
& p=\alpha^{2} f(t) t^{-2}\left[b(t) x^{\lambda}+Q(t)\right],
\end{aligned}
$$

where $\alpha$ and $\lambda$ are arbitrary constants, $a(t)$ is related to $f(t)$ by

$$
a(t)=\frac{\alpha \lambda-t f_{t} / f}{\lambda+1} .
$$

Moreover, $Q(t)$ is a new arbitrary function of $t$ and the function

$$
b(t)=\frac{(\lambda+1)}{\lambda \alpha^{2}}\left[-a^{2}+a-t a_{t}\right]
$$

McVittie [1] considered the nonsingular case with $Q(t)=0$ and $\lambda>2$ and imposed the particle adiabacy condition (10). He thus obtained the following equation for $a(t)$,

$$
a_{z z}+[-3+(3 \gamma+1) a] a_{z}+[2-(3 \gamma-1) a](1-a) a=0,
$$

where $z=\log t$. Equation (14) was transformed to an Abel equation which, however, was left unsolved. Here, by using the transformation

$$
a=\frac{t j_{t}}{j}, \quad t=e^{z},
$$

in (14), we get a third-order nonlinear ODE

$$
j j_{t t t}+(3 \gamma-2) j_{t} j_{t t}=0,
$$

which for $\gamma \neq 1$ can be integrated to yield

$$
j(t)=C t^{\frac{2}{3(1-\gamma)}},
$$

where $C$ is an arbitrary constant. 
Case B:

McVittie [1] assumed that $g=-1$ yielding $f=t^{-\alpha}$. So, he wrote the solution

$$
\begin{aligned}
& q=\frac{\alpha r}{t}, \\
& \rho=t^{-3 \alpha} F(x), \\
& p=\alpha^{2} t^{-\alpha-2}[G(x)+Q(t)],
\end{aligned}
$$

where $Q(t)$ is again an arbitrary function while

$$
\begin{aligned}
& F(x)=x^{-2}\left(x^{2} w^{\prime}\right)^{\prime}, \\
& G(x)=\frac{\alpha+1}{\alpha} w+\left(2+\frac{1}{\alpha} x w^{\prime}+x\left(x w^{\prime}\right)\right)^{\prime}-x u-2 \int u d x .
\end{aligned}
$$

We remark that the solutions (18) and (19) (see Equations (25) and (26) of Keller [3]) can easily be obtained by assuming that

$$
x^{-2}\left(x^{2} w^{\prime}\right)^{\prime}=-\frac{F(x)^{\prime}}{\lambda x},
$$

or, with $c_{1}, c_{2}$ arbitrary,

$$
w=c_{2}-c_{1}-\frac{1}{x} \int^{x} F(y) d y,
$$

where $F$ is an arbitrary function of $x$. McVittie [1] showed that the imposition of the adiabatic condition (10) leads to two distinct cases, namely,

$$
\alpha=\frac{2}{(3 \gamma-1)}, \quad Q=\text { constant },
$$

or, with $\epsilon$ and $\delta$ constants,

$$
Q=-\epsilon+\delta t^{-\alpha(3 \gamma-1)+2}
$$

\section{Solutions with nonlinear particle velocity}

Note that all the solutions found by McVittie [1] have linear particle velocity with respect to $r$ and need to be generalized to be applicable to more realistic situations. This is what we attempt to accomplish in the following. To that end, it is convenient to introduce

$$
a(x)=\frac{(1-g) w_{x}+x w_{x x}}{2 w_{x}+x w_{x x}} .
$$

From the expression for $q$ in (6), we get

$$
q=\alpha r t^{-1} a(x), \quad x=r t^{-\alpha} .
$$


We assume $g$ to be a constant so that $f(t)$ is determined from the first equation of (9) as

$$
f(t)=t^{\alpha g}
$$

We express $q, \rho$, and $p$ in terms of $a$ so that Equations (1) and (2) are satisfied. We then impose the adiabatic condition (3). This leads to a second-order ODE for $a$, which may be reduced to first-order. Two cases are treated: $a=1$ and $a(x) \neq 1$ for any $x$. We observe from (20) that the case $=1$ is tantamount to assuming that $g+1=0$. This special case was treated by McVittie [1]. We briefly treat this case first.

From (9) we get

$$
v=x^{2} w_{x}, \quad v_{x}=2 x w_{x}+x^{2} w_{x x}, \quad u=2 w_{x}+x w_{x x}
$$

and

$$
2 \int^{x} x^{1+g} u d x=2 \int^{x} w_{x}+\left(x w_{x}\right)_{x} d x=2 w+2 x w_{x}+Q(t),
$$

where $Q(t)$ is a function of $t$. Using these expressions in (6)-(8), we have

$$
\begin{aligned}
& q=\alpha t^{\alpha-1} x, \\
& \rho=t^{-3 \alpha} \frac{2 w_{x}+x w_{x x}}{x}, \\
& p=p_{0}+\alpha(1-\alpha) t^{-2-\alpha}\left(w+x w_{x}+Q(t)\right) .
\end{aligned}
$$

The adiabatic condition (10) leads to

$$
\frac{\alpha(3 \gamma-1)-2}{t}+\frac{Q^{\prime}(t)}{w+x w_{x}+Q(t)}=0,
$$

which gives either $Q^{\prime}(t)=0$ and $\alpha=\frac{2}{3 \gamma-1}$, or $(x w)_{x}=c_{0}$, a constant, leading to $w=c_{0}+\frac{c_{1}}{x}, Q(t)=-c_{0}+c_{2} t^{2-\alpha(3 \gamma-1)}$, where $c_{0}, c_{1}$, and $c_{2}$ are constants. Thus, we have two sets of solutions, which satisfy the adiabatic condition. The first solution contains an arbitrary function $w$ of $x=r t^{-\alpha}$ :

$$
\begin{aligned}
& q=\frac{2}{3 \gamma-1} t^{\frac{2}{3 \gamma-1}-1} x, \\
& \rho=t^{\frac{-6}{3 \gamma-1}} \frac{2 w_{x}+x w_{x x}}{x}, \\
& p=p_{0}+\frac{2}{3 \gamma-1}\left(1-\frac{2}{3 \gamma-1}\right) t^{-2-\frac{2}{3 \gamma-1}}\left((x w)_{x}+c_{0}\right),
\end{aligned}
$$


where $c_{0}$ is a constant. The second solution is rather special:

$$
\begin{aligned}
& q=\alpha t^{\alpha-1} x, \\
& \rho=0, \\
& p=p_{0}+\alpha c_{1}(1-\alpha) t^{-3 \alpha \gamma},
\end{aligned}
$$

where $c_{1}$ is a constant.

Now we consider the more general solution for which the function $a(x) \neq 1$ is arbitrary. We observe from (20) that the functions $a$ and $w$ are related by the ODE

$$
w^{\prime \prime}=A(x) w^{\prime}
$$

where $w^{\prime}=w_{x}$ and

$$
A(x)=\frac{1-g-2 a}{x(a-1)} .
$$

Solving (23), we obtain

$$
w^{\prime}(x)=c_{0} e^{\int^{x} A(z) d z} .
$$

A second integration gives

$$
w(x)=c_{1}+c_{0} \int^{x} e^{\int^{y} A(z) d z} d y .
$$

Using (25) in (6)-(8), we get a solution of the system (1) and (2), which depends on an arbitrary function $a(x) \neq 1, x=r t^{-\alpha}$, namely,

$$
\begin{aligned}
& q(x, t)=\alpha t^{\alpha-1} Q(x), \\
& \rho(x, t)=-c_{0}(1+g) t^{\alpha(g-2)} R(x), \\
& p(x, t)=p_{0}+\alpha^{2} t^{\alpha g-2} P(x),
\end{aligned}
$$

where

$$
\begin{gathered}
Q(x)=x a(x), \\
R(x)=\frac{1}{x(a(x)-1)} e^{\int^{x} A(z) d z} \\
\left.P(x)=g\left(g-\frac{1}{\alpha}\right) c_{1}+c_{0}\left[\left(\frac{1}{\alpha}-g\right)+(1+g) a(x)\right)\right] x e^{\int^{x} A(z) d z} \\
+c_{0} \int^{x}\left[2(1+g) \frac{a^{2}(y)}{a(y)-1}+\left(g^{2}-\frac{g}{\alpha}\right)\right] e^{\int^{y} A(z) d z} d y .
\end{gathered}
$$


Now, we find the function $a(x)$ such that the solution (26)-(31) also satisfies the particle adiabacy condition (10).

For any function $F(x)$ with $x=r t^{-\alpha}$, we have

$$
\left(\partial_{t}+q \partial_{r}\right) F(x)=\frac{x}{t} \alpha(a-1) F^{\prime}(x) .
$$

Thus, on using (26)-(31) in the adiabatic condition (10), we get

$$
\beta+\alpha x(a-1)\left(\frac{P^{\prime}}{P}-\gamma \frac{R^{\prime}}{R}\right)=0,
$$

where

$$
\beta=\alpha g(1-\gamma)+2(\alpha \gamma-1)
$$

We choose $c_{0}=1$ and $c_{1}=0$ in (29)-(31). A simple calculation shows that

$$
\frac{R^{\prime}}{R}=\frac{2-g-3 a-x a^{\prime}}{x(a-1)} \text {. }
$$

Using (34) in (32), we have

$$
\frac{P^{\prime}}{P}=\frac{2-\alpha g-3 \alpha \gamma a-\alpha \gamma x a^{\prime}}{\alpha x(a-1)} .
$$

First, we consider the case $2-\alpha g-3 \alpha \gamma a-\alpha \gamma x a^{\prime} \neq 0$. Here, we may write (35) in the form

$$
P=C P^{\prime}
$$

where

$$
C=\frac{\alpha x(a-1)}{2-\alpha g-3 \alpha \gamma a-\alpha \gamma x a^{\prime}} .
$$

Differentiating (36), we get

$$
\left(1-C^{\prime}\right) P^{\prime}=C P^{\prime \prime}
$$

From (31), we have

$$
P^{\prime}=(1+g)\left[\left(1-\frac{1}{\alpha}\right)+\frac{1-\frac{1}{\alpha}}{a-1}+a+x a^{\prime}\right] e^{\int^{x} A(z) d z}
$$

and

$$
\begin{aligned}
P^{\prime \prime}= & \frac{(1+g)}{x(a-1)^{2}} e^{\int^{x} A(z) d z} \times\left[x^{2} a^{2} a^{\prime \prime}-2 x^{2} a a^{\prime \prime}+x^{2} a^{\prime \prime}-(1+g) x a a^{\prime}\right. \\
& \left.-2 a^{3}+\left(1+\frac{2}{\alpha}-g\right) a^{2}+\left(\frac{1}{\alpha}+g\right) x a^{\prime}-\frac{1}{\alpha}(1-g) a\right] .
\end{aligned}
$$


Substituting (39) and (40) into (38) we get

$$
\begin{aligned}
(1- & \left.C^{\prime}\right)\left[\left(1-\frac{1}{\alpha}\right)\left(\frac{a}{a-1}\right)+a+x a^{\prime}\right] \\
= & \frac{C}{x(a-1)^{2}} \times\left[x^{2} a^{2} a^{\prime \prime}-2 x^{2} a a^{\prime \prime}+x^{2} a^{\prime \prime}-(1+g) x a a^{\prime}-2 a^{3}\right. \\
& \left.+\left(1+\frac{2}{\alpha}-g\right) a^{2}+\left(g+\frac{1}{\alpha}\right) x a^{\prime}-\frac{1}{\alpha}(1-g) a\right] .
\end{aligned}
$$

Using (37) in (41), we obtain

$$
\begin{aligned}
{\left[1-C^{\prime}\right]\left[\frac{2}{\alpha}-g-\gamma\left(3 a+x a^{\prime}\right)\right]\left[a^{2}-\frac{a}{\alpha}+x a a^{\prime}-x a^{\prime}\right] } \\
=\left[x^{2} a^{2} a^{\prime \prime}-2 x^{2} a a^{\prime \prime}+x^{2} a^{\prime \prime}-(1+g) x a a^{\prime}-2 a^{3}\right. \\
\left.+\left(1+\frac{2}{\alpha}-g\right) a^{2}+\left(g+\frac{1}{\alpha}\right) x a^{\prime}-\frac{1}{\alpha}(1-g) a\right] .
\end{aligned}
$$

Differentiating (37), we get

$$
\begin{aligned}
& C^{\prime}= \\
& \frac{\left(g-\frac{2}{\alpha}\right)+\left(\frac{2}{\alpha}-g+3 \gamma\right) a+\left(\frac{2}{\alpha}-g-3 \gamma\right) x a^{\prime}-\gamma x^{2} a^{\prime 2}+\gamma x^{2} a a^{\prime \prime}-3 \gamma a^{2}-\gamma x^{2} a^{\prime \prime}}{\left(\frac{2}{\alpha}-g-\gamma\left(3 a+x a^{\prime}\right)\right)^{2}} .
\end{aligned}
$$

Substituting $C^{\prime}$ from (43) into (42), we obtain a complicated ODE for $a=a(x)$ :

$$
\begin{aligned}
& {\left[\left(\frac{2}{\alpha}-g-\gamma\left(3 a+x a^{\prime}\right)\right)^{2}-\left(g-\frac{2}{\alpha}\right)-\left(\frac{2}{\alpha}-g+3 \gamma\right) a\right.} \\
& \left.\quad-\left(\frac{2}{\alpha}-g-3 \gamma\right) x a^{\prime}+\gamma x^{2} a^{\prime 2}-\gamma x^{2} a a^{\prime \prime}+3 \gamma a^{2}+\gamma x^{2} a^{\prime \prime}\right] \\
& \quad \times\left[a^{2}-\frac{a}{\alpha}+x a a^{\prime}-x a^{\prime}\right]-\left[\left(\frac{2}{\alpha}-g\right)-\gamma\left(3 a+x a^{\prime}\right)\right] \\
& \quad \times\left[x^{2} a^{2} a^{\prime \prime}-2 x^{2} a a^{\prime \prime}+x^{2} a^{\prime \prime}-(1+g) x a a^{\prime}-2 a^{3}\right. \\
& \left.\quad+\left(1+\frac{2}{\alpha}-g\right) a^{2}+\left(g+\frac{1}{\alpha}\right) x a^{\prime}-\frac{1}{\alpha}(1-g) a\right]=0 .
\end{aligned}
$$

Thus $q, \rho$, and $p$ given by (26)-(28) and (29)-(31) constitute an exact solution of the system (1) and (2) for arbitrary $a(x) \neq 1$. This solution also satisfies the 
adiabatic condition (10) in the region $2-\alpha g-3 \alpha \gamma a-\alpha \gamma x a^{\prime} \neq 0$ if $a$ satisfies the ODE (44).

We observe that we may also work in terms of the arbitrary function $w$ as in McVittie [1]. Then, we get a third-order ODE for $w^{\prime}$. This ODE may easily be obtained by substituting

$$
a(x)=\frac{(1-g) w^{\prime}+x w^{\prime \prime}}{2 w^{\prime}+x w^{\prime \prime}}
$$

in the second-order ODE (44) for $a$. The former, however, does not seem to be more amenable to analysis.

Next, we consider the region $2-\alpha g-3 \alpha \gamma a-\alpha \gamma x a^{\prime}=0$. In this case, $P^{\prime}$ is given by (39); $P^{\prime}$ is also zero in view of (35). Because we assume that $(1+g) \neq 0$, we get from (39)

$$
\left(1-\frac{1}{\alpha}\right) \frac{a}{a-1}+a+x a^{\prime}=0 .
$$

In the region under consideration,

$$
x a^{\prime}=\frac{2-\alpha g-3 \alpha \gamma}{\alpha \gamma} .
$$

From (46) and (47), we get a quadratic equation for $a$ with constant coefficients, showing that $a$ is a constant. As an example, let $g=\frac{2}{\alpha}$; clearly, $2-\alpha g-$ $3 \alpha \gamma a-\alpha \gamma x a^{\prime}=0$ is satisfied by the nonconstant function,

$$
a(x)=\lambda_{0} x^{-3},
$$

and so the solution of the system (1)-(2) given by (26)-(31) for the function (47) does not satisfy the adiabatic condition.

Now we show that Equation (44) may be solved locally. If we set $x=e^{z}$, then $x a^{\prime}=a_{z}, x^{2} a^{\prime \prime}=a_{z z}-a_{z}$, and Equation (44) reduces to the autonomous ODE,

$$
\begin{aligned}
& {\left[\left(\frac{2}{\alpha}-g-\gamma\left(3 a+a_{z}\right)\right)^{2}-\left(g-\frac{2}{\alpha}\right)-\left(\frac{2}{\alpha}-g+3 \gamma\right) a\right.} \\
& \left.\quad-\left(\frac{2}{\alpha}-g-3 \gamma\right) a_{z}+\gamma a_{z}^{2}-\gamma a\left(a_{z z}-a_{z}\right)+3 \gamma a^{2}+\gamma\left(a_{z z}-a_{z}\right)\right] \\
& \quad \times\left[a^{2}-\frac{a}{\alpha}+a a_{z}-a_{z}\right]-\left[\left(\frac{2}{\alpha}-g\right)-\gamma\left(3 a+a_{z}\right)\right] \\
& \quad \times\left[a^{2}\left(a_{z z}-a_{z}\right)-2 a\left(a_{z z}-a_{z}\right)+a_{z z}-a_{z}-(1+g) a a_{z}-2 a^{3}\right. \\
& \left.\quad+\left(1+\frac{2}{\alpha}-g\right) a^{2}+\left(g+\frac{1}{\alpha}\right) a_{z}-\frac{1}{\alpha}(1-g) a\right]=0 .
\end{aligned}
$$


Equation (48) may be reduced to a first-order equation in the variable $p=\frac{d a}{d z}$ with $a$ as the independent variable. Thus, we have

$$
\begin{aligned}
& {\left[\left(\frac{2}{\alpha}-g-\gamma(3 a+p)\right)^{2}-\left(g-\frac{2}{\alpha}\right)-\left(\frac{2}{\alpha}-g+3 \gamma\right) a\right.} \\
& \left.\quad-\left(\frac{2}{\alpha}-g-3 \gamma\right) p+\gamma p^{2}-\gamma a p\left(\frac{d p}{d a}-1\right)+3 \gamma a^{2}+\gamma p\left(\frac{d p}{d a}-1\right)\right] \\
& \quad \times\left[a^{2}-\frac{a}{\alpha}+p(a-1)\right]-\left[\left(\frac{2}{\alpha}-g\right)-\gamma(3 a+p)\right] \\
& \quad \times\left[(a-1)^{2} p\left(\frac{d p}{d a}-1\right)-p-(1+g) a p-2 a^{3}+\left(1+\frac{2}{\alpha}-g\right) a^{2}\right. \\
& \left.\quad+\left(g+\frac{1}{\alpha}\right) p-\frac{1}{\alpha}(1-g) a\right]=0 .
\end{aligned}
$$

The coefficient of $\frac{d p}{d a}$ can easily be found to be

$$
p(a-1)\left(2 \gamma a^{2}+\left(g+\frac{\gamma-2}{\alpha}-3 \gamma\right) a-\left(g-\frac{2}{\alpha}\right)\right) .
$$

As we are interested in the case where $a$ is not a constant, the coefficient (50) is not zero and hence (49) is a genuine first-order equation linear in $\frac{d p}{d a}$. By the local existence theory of ODEs (see Coddington and Levinson [7]), there exists solution of (49) in a neighborhood of any point $a=a_{0}$ with the condition that $p\left(a_{0}\right) \neq 0$. Thus, the existence of a large class of solutions to (1) and (2) given by (26)-(31) and satisfying the adiabatic condition (locally) follows. To obtain the local structure of the solutions, we observe that (44) can be written in the form $a^{\prime \prime}=F\left(x, a, a^{\prime}\right)$, where $F$ is analytic in its arguments except at $x=0, a=1, \alpha_{1}, \alpha_{2}$, or $a^{\prime}=0$, where $\alpha_{1}$ and $\alpha_{2}$ are roots of the equation $2 \gamma \xi^{2}+\left(g+\frac{\gamma-2}{\alpha}-3 \gamma\right) \xi-\left(g-\frac{2}{\alpha}\right)=0$. By an application of Cauchy-Kowalewski theorem to Equation (44), we get the following form of solution for $x$ in a neighborhood of $x_{0}>0$ :

$$
a(x)=\sum_{0}^{\infty} \lambda_{k}\left(x-x_{0}\right)^{k}
$$

Another form of solution for (44) is obtained on applying Cauchy-Kowalewski theorem to the transformed Equation (48), namely,

$$
a(x)=\sum_{0}^{\infty} \lambda_{k}\left(\log \left(\frac{x}{x_{0}}\right)\right)^{k}
$$

for $\log \left(x / x_{0}\right)$ small. In (51) and (52), $\lambda_{0}$ can not assume values $1, \alpha_{1}$, or $\alpha_{2}$; $\lambda_{1} \neq 0$ may be prescribed arbitrarily. All other coefficients $\lambda_{k}, k=2,3,4, \ldots$. 
are determined in terms of $\lambda_{0}$ and $\lambda_{1}$ and the parameters $\alpha, g$, and $\gamma$ appearing in Equation (44). Our concern here is to find the exact solutions and some of their qualitative properties. More generally, one should solve Equation (44) numerically subject to appropriate initial conditions, say, at the shock. In the next section, we consider a special nonlinear function $a(x)$ and investigate when it may satisfy the adiabatic condition exactly.

\section{Special nonlinear velocity profile: $a(x)=\lambda_{0} x^{\mu}+\lambda_{1}$}

Motivated by the approximate similarity solution of Taylor [2] for the point explosion, we let

$$
a(x)=\lambda_{0} x^{\mu}+\lambda_{1},
$$

where $\lambda_{0}$ and $\lambda_{1}$ are constants. First, we find exact solutions (26)-(28) and (29)-(31), which correspond to (53). Then, we show that for $\lambda_{1}=0$ and special choices of the parameters $\mu, \alpha$, and $g$, the adiabatic condition (10) is also satisfied.

For $a(x)$ defined by (53), (24) gives

$$
A(x)=\frac{1-g-2 \lambda_{0} x^{\mu}-2 \lambda_{1}}{x\left(\lambda_{0} x^{\mu}+\lambda_{1}-1\right)},
$$

which may be written as

$$
A(x)=-\frac{(1+g)}{\lambda_{0}} x^{-(1+\mu)}-2 x^{-1}, \quad \lambda_{1}=1
$$

and

$$
A(x)=\frac{\left(1-g-2 \lambda_{1}\right)}{\lambda_{1}-1} \frac{1}{x}-\frac{(1+g)}{\lambda_{1}-1} \frac{\lambda_{0} x^{\mu-1}}{\lambda_{0} x^{\mu}+\lambda_{1}-1}, \quad \lambda_{1} \neq 1 .
$$

Integrating (55) and (56), we get

$$
\int^{x} A(z) d z=\frac{(1+g)}{\lambda_{0} \mu} x^{-\mu}-2 \log x+A_{0}, \quad \lambda_{1}=1
$$

and

$$
\int^{x} A(z) d z=\log A_{0}+\log \frac{\left(\lambda_{0} x^{\mu}+\lambda_{1}-1\right)^{\frac{1+g}{\mu\left(1-\lambda_{1}\right)}}}{x^{\frac{1-g-2 \lambda_{1}}{1-\lambda_{1}}}}, \quad \lambda_{1} \neq 1 .
$$

Substituting (55) and (56) in (26)-(31), we get the following forms of the solution for $\lambda_{1}=1$ and $\lambda_{1} \neq 1$. 
(i) $\lambda_{1}=1$.

$$
\begin{aligned}
& q(x, t)=\alpha t^{\alpha-1} x\left(\lambda_{0} x^{\mu}+1\right), \\
& \rho(x, t)=-c_{0} A_{0} \lambda_{0}^{-1}(1+g) t^{\alpha(g-2)} x^{-(\mu+3)} e^{\frac{(1+g)}{\lambda_{0} \mu} x^{-\mu}}, \\
& p(x, t)=p_{0}+\alpha^{2} t^{\alpha g-2} P(x),
\end{aligned}
$$

where

$$
\begin{aligned}
P(x)= & g\left(g-\frac{1}{\alpha}\right) c_{1}+c_{0} A_{0}\left(\left(\frac{1}{\alpha}-g\right)+(1+g)\left(\lambda_{0} x^{\mu}+1\right)\right) x^{-1} e^{\frac{(1+g)}{\lambda_{0} \mu} x^{-\mu}} \\
& +c_{0} A_{0} \int^{x}\left(\frac{2(1+g)\left(\lambda_{0} s^{\mu}+\lambda_{1}\right)^{2}}{\lambda_{0} s^{\mu}}+\left(g^{2}-\frac{g}{\alpha}\right)\right) s^{-2} e^{\frac{1+g}{\lambda_{0} \mu} s^{-\mu}} d s .
\end{aligned}
$$

(ii) $\lambda_{1} \neq 1$.

$$
\begin{aligned}
& q(x, t)=\alpha t^{\alpha-1} x\left(\lambda_{0} x^{\mu}+\lambda_{1}\right) \\
& \rho(x, t)=-c_{0} A_{0}(1+g) t^{\alpha(g-2)} \frac{\left(\lambda_{0} x^{\mu}+\lambda_{1}-1\right)^{\frac{1+g}{\mu\left(1-\lambda_{1}\right)}-1}}{x^{1+\frac{1-g-2 \lambda_{1}}{1-\lambda_{1}}}} \\
& p(x, t)=p_{0}+\alpha^{2} t^{\alpha g-2} P(x),
\end{aligned}
$$

where

$$
\begin{aligned}
P(x)= & g\left(g-\frac{1}{\alpha}\right) c_{1} \\
& +c_{0} A_{0}\left[\left(\frac{1}{\alpha}-g\right)+(1+g)\left(\lambda_{0} x^{\mu}+\lambda_{1}\right) \frac{\left(\lambda_{0} x^{\mu}+\lambda_{1}-1\right)^{\frac{1+g}{\mu\left(1-\lambda_{1}\right)}}}{x^{-1+\frac{\left(1-g-2 \lambda_{1}\right)}{1-\lambda_{1}}}}\right] \\
& +c_{0} A_{0} \int^{x}\left[2(1+g) \frac{\left(\lambda_{0} s^{\mu}+\lambda_{1}\right)^{2}}{\left(\lambda_{0} s^{\mu}+\lambda_{1}-1\right)}+\left(g^{2}-\frac{g}{\alpha}\right)\right] \\
& \times \frac{\left(\lambda_{0} s^{\mu}+\lambda_{1}-1\right)^{\frac{1+g}{\mu\left(1-\lambda_{1}\right)}}}{s^{\frac{1-g-2 \lambda_{1}}{1-\lambda_{1}}}} d s .
\end{aligned}
$$

Now, we attempt to find solutions also which satisfy the adiabatic condition (10). When $g=\frac{2}{\alpha}$, Equation (44) becomes 


$$
\begin{aligned}
& {\left[\gamma^{2}\left(3 a+x a^{\prime}\right)^{2}-3 \gamma a+3 \gamma x a^{\prime}+\gamma x^{2} a^{\prime 2}-\gamma x^{2} a a^{\prime \prime}+3 \gamma a^{2}+\gamma x^{2} a^{\prime \prime}\right]} \\
& \quad \times\left[\left(a^{2}-\frac{a}{\alpha}+x a a^{\prime}-x a^{\prime}\right)+\gamma\left(3 a+x a^{\prime}\right)\right] \times\left[x^{2} a^{2} a^{\prime \prime}-2 x^{2} a a^{\prime \prime}+x^{2} a^{\prime \prime}\right. \\
& \left.\quad-(1+g) x a a^{\prime}-2 a^{3}+a^{2}+\left(g+\frac{1}{\alpha}\right) x a^{\prime}-\frac{1}{\alpha}(1-g) a\right]=0
\end{aligned}
$$

For $a=\lambda_{0} x^{\nu}+\lambda_{1},(63)$ becomes

$$
\begin{aligned}
& {\left[\gamma^{2}(3+\mu)^{2} a^{2}-2 \gamma^{2} \mu \lambda_{1}(3+\mu) a+\gamma^{2} \mu^{2} \lambda_{1}^{2}-3 \gamma a+3 \gamma \mu\left(a-\lambda_{1}\right)\right.} \\
& \left.\quad+\gamma \mu^{2}\left(a-\lambda_{1}\right)^{2}-\gamma \mu(\mu-1) a\left(a-\lambda_{1}\right)+3 \gamma a^{2}+\gamma \mu(\mu-1)\left(a-\lambda_{1}\right)\right] \\
& \quad \times\left[a^{2}-\frac{a}{\alpha}+\mu a\left(a-\lambda_{1}\right)-\mu\left(a-\lambda_{1}\right)\right]+\gamma\left[(3+\mu) a-\mu \lambda_{1}\right] \\
& \quad \times\left[\mu(\mu-1) a^{2}\left(a-\lambda_{1}\right)-2 \mu(\mu-1) a\left(a-\lambda_{1}\right)+\mu(\mu-1)\left(a-\lambda_{1}\right)\right. \\
& \left.\quad-(1+g) \mu a\left(a-\lambda_{1}\right)-2 a^{3}+a^{2}+\left(g+\frac{1}{\alpha}\right) \mu\left(a-\lambda_{1}\right)-\frac{(1-g)}{\alpha} a\right]=0,
\end{aligned}
$$

which is a polynomial in $a$ of degree 4 . Because the polynomial is identically 0 , we get five algebraic equations connecting four parameters $\lambda_{1}, \mu, \alpha$, and $\gamma$ :

$$
\begin{aligned}
& \left(\gamma^{2}(3+\mu)^{2}+\gamma \mu^{2}-\gamma \mu(\mu-1)+3 \gamma\right)(1+\mu) \\
& \quad+\gamma(3+\mu)(\mu(\mu-1)-2)=0, \\
& {\left[\gamma^{2}(3+\mu)^{2}+\gamma \mu^{2}-\gamma \mu(\mu-1)+3 \gamma\right] \times\left[\frac{1}{\alpha}+\mu \lambda_{1}+\mu\right]} \\
& \quad+\left[\lambda_{1} \mu(\mu-1)+2 \mu(\mu-1)+(1+g) \mu-1\right] \times[\gamma(3+\mu)] \\
& \quad+\gamma \mu \lambda_{1}(\mu(\mu-1)-2)(1+\mu)\left(2 \gamma^{2} \mu \lambda_{1}(3+\mu)\right. \\
& \left.\quad+3 \gamma(1-\mu)+2 \gamma \mu^{2} \lambda_{1}-\left(\lambda_{1}+1\right) \gamma \mu(\mu-1)\right)=0, \\
& \left.\mu \lambda_{1}\left[\gamma^{2}(3+\mu)^{2}+\gamma \mu^{2}-\gamma \mu(\mu-1)\right]+3 \gamma\right)-\left[\frac{1}{\alpha}+\mu \lambda_{1}+\mu\right] \\
& \quad \times\left[\gamma \mu(\mu-1)+\lambda_{1} \gamma \mu(\mu-1)-2 \lambda_{1} \gamma \mu^{2}+3 \gamma(\mu-1)-2 \gamma^{2} \mu \lambda_{1}(3+\mu)\right] \\
& \quad+(1+\mu)\left(\gamma^{2} \mu^{2} \lambda_{1}^{2}-3 \gamma \mu \lambda_{1}+\gamma \mu^{2} \lambda_{1}^{2}-\gamma \mu(\mu-1) \lambda_{1}\right) \\
& \quad+\gamma \mu \lambda_{1}\left[\lambda_{1} \mu(\mu-1)+2 \mu(\mu-1)+(1+g) \mu+1\right] \\
& \quad+\gamma(3+\mu)\left[2 \lambda_{1} \mu(\mu-1)+\mu(\mu-1)+\mu \lambda_{1}(1+g)\right] \\
& \quad+\left(g+\frac{1}{\alpha}\right) \mu-\frac{(1-g)}{\alpha}=0,
\end{aligned}
$$




$$
\begin{aligned}
& \mu \lambda_{1}\left(2 \gamma^{2} \mu \lambda_{1}(3+\mu)+3 \gamma-3 \gamma \mu+2 \lambda_{1} \gamma \mu^{2}-\gamma \mu(\mu-1) \lambda_{1}-\gamma \mu(\mu-1)\right) \\
& +\left(\frac{1}{\alpha}+\mu \lambda_{1}+\mu\right)\left(\gamma^{2} \mu^{2} \lambda_{1}^{2}-3 \gamma \mu \lambda_{1}+\gamma \mu^{2} \lambda_{1}^{2}-\gamma \mu(\mu-1) \lambda_{1}\right) \\
& +\gamma(3+\mu)\left[\lambda_{1} \mu(\mu-1)+\lambda_{1} \mu\left(g+\frac{1}{\alpha}\right)\right] \\
& +\gamma \mu \lambda_{1}\left[2 \lambda_{1} \mu(\mu-1)+\mu(\mu-1)+\lambda_{1} \mu(1+g)\right. \\
& \left.+\left(g+\frac{1}{\alpha}\right) \mu-\frac{(1-g)}{\alpha}\right]=0 \\
& \mu \lambda_{1}\left(\gamma^{2} \mu^{2} \lambda_{1}^{2}-3 \gamma \mu \lambda_{1}+\gamma \mu^{2} \lambda_{1}^{2}-\gamma \mu(\mu-1) \lambda_{1}\right) \\
& +\gamma \mu \lambda_{1}\left[\lambda_{1} \mu(\mu-1)+\lambda_{1} \mu\left(g+\frac{1}{\alpha}\right)\right]=0
\end{aligned}
$$

Equation (64) may be written as

$$
\left(\gamma^{2}(3+\mu)^{2}+\gamma(3+\mu)\right)(1+\mu)+\gamma(3+\mu)(\mu+1)(\mu-2)=0 .
$$

Canceling out $\gamma(3+\mu)$ and rewriting this equation, we get $\gamma(\mu+1) \times$ $(3+\mu)(\gamma(3+\mu)+\mu-1)=0$. Thus, the possible roots for $\mu$ are

$$
\mu=-3, \quad \mu=-1, \quad \mu=\frac{1-3 \gamma}{1+\gamma} .
$$

Now, we restrict our attention to the special case, $\lambda_{1}=0$. In this case, (67) and (68) are trivially satisfied; therefore, we need to treat Equations (65) and (66) only. Further, the root $\mu=-3$ is ruled out by the discussions in the previous section since, for $a$ corresponding to this, the expression $2-\alpha g-$ $\alpha \gamma\left(3 a+x a^{\prime}\right)$ vanishes identically.

Equation (65) can be written as

$$
\begin{aligned}
& {\left[\gamma^{2}(3+\mu)^{2}+\gamma(3+\mu)\right] \times\left[\frac{1}{\alpha}+\mu\right]} \\
& \quad+\left[2 \mu(\mu-1)+1+\frac{2}{\alpha} \mu-1+(1+\mu)(1-\mu)\right] \times[\gamma(3+\mu)]=0 .
\end{aligned}
$$

Canceling out $\gamma(3+\mu)$, we get

$$
\begin{aligned}
& {[\gamma(3+\mu)+1] \times\left[\frac{1}{\alpha}+\mu\right]+2 \mu(\mu-1)} \\
& \quad+\left(1+\frac{2}{\alpha} \mu-1+(1+\mu)(1-\mu)\right)=0 .
\end{aligned}
$$


Setting $\lambda_{1}=0$, and $g=\frac{2}{\alpha}$, we obtain from (66)

$-\gamma(3+\mu)\left(\frac{1}{\alpha}+\mu\right)(\mu-1)+\gamma(3+\mu)\left[\left(\mu(\mu-1)+\frac{3}{\alpha} \mu-\frac{1}{\alpha}\left(1-\frac{2}{\alpha}\right)\right]=0\right.$.

Canceling out $\gamma(3+\mu)$, we get

$$
-(\mu-1)(1 \alpha+\mu)+\mu(\mu-1)+\frac{3 \mu}{\alpha}-\frac{1}{\alpha}\left(1-\frac{2}{\alpha}\right)=0
$$

Solving (71), we have

$$
\mu=-\frac{1}{\alpha}
$$

We must show that $\mu$ given by (69) satisfies (70) and (72). We first consider the case $\mu=-1$. From (72), we have $\alpha=1$. With $\mu=-1$ and $\alpha=1$, (70) is satisfied for all $\gamma$. Thus, we have a set of solutions of the system (64)-(68):

$$
\mu=-1, \quad \alpha=1, \quad g=2, \quad \gamma \neq 0 .
$$

When $\mu=\frac{1-3 \gamma}{1+\gamma}$, (70) gives $\gamma=1$; this is not admissible because we have assumed that $\gamma>1$. In conclusion, for $\gamma>1$ and $\alpha=1, g=2$, and $a=$ $\lambda_{0} x^{-1}$ in (61) and (62) gives a solution of the system (1) and (2), which satisfies the adiabatic condition (10).

It is easily seen that for general $a(x)$ of the form (53), the possible values of $\mu$ for which solution (59) satisfies the adiabatic condition are $-3,-1$, or $\frac{1-3 \gamma}{1+\gamma}$. As $\gamma>1$, the only possible value of $\mu$ for which the velocity $q=\alpha t^{\alpha-1} x a(x)$ is finite is $\mu=-1$. This value is quite different from $\mu>2$ assumed by Taylor [2]. Thus, our analysis shows that there is no exact adiabatic solution with the form of velocity chosen by Taylor [2]. The form of adiabatic solution with nonlinear velocity is far more complex.

\section{Concluding remarks}

Taylor [2], in his study of formation of blast waves from an intense explosion, considered the compressible flow Equations (1) and (2) with spherical symmetry along with the adiabatic condition (10). Similarity assumptions for an expanding blast wave of constant energy led to the following form of the velocity $q$, density $\rho$, and pressure $p$ :

$$
q(x, t)=t^{-3 / 5} Q(x), \quad \rho(x, t)=R(x), \quad p(x, t)=t^{-6 / 5} P(x),
$$

where $x=r t^{-2 / 5}$. Taylor [2] considered the special case $Q(x)=\lambda_{0} x^{n}+$ $x / \gamma$ and found approximate solutions for $\rho$ and $p$ under various physical 
assumptions. These solutions motivated us to see exact solutions of the form (59)-(62). Taylor [8] considered Equations (1) and (2) along with the condition (10) and obtained the solution in the form (74) implicitly. At the shock front, $R=k t^{2 / 5}, \rho$, and $p$ were expressed in terms of $q ; q$ itself was given by an implicit relation. All the adiabatic solutions constructed by McVittie [1] have particle velocity $q$ linear in $r$ (see Equation (8)). We have given the solutions (26)-(31) of (1) and (2) explicitly in terms of a function $a(x)$ of the similarity variable $x=r t^{-\alpha}$. McVittie's result corresponds to the case $a=1$. We assume that $a \neq 1$ and show that the solutions (26)-(31) satisfies the adiabatic condition (10) provided $a(x)$ satisfies (44), a second-order nonlinear ODE; the latter is reduced to an equation of first-order and hence analyzed. The local existence of solution of (44) is demonstrated. We show that for any $x_{0}>0$, there is a small neighborhood of $x_{0}$, where (44) has analytic solutions of the form

$$
a(x)=\sum_{0}^{\infty} \lambda_{k}\left(x-x_{0}\right)^{k} .
$$

We have also shown the existence of local solutions of the forms

$$
a(x)=\sum_{0}^{\infty} \lambda_{k}\left(\log \left(\frac{x}{x_{0}}\right)\right)^{k}
$$

for $\log \left(x / x_{0}\right)$ small. These solutions are a two parameter family each, provided that $\lambda_{0}$ does not assume the values $1, \alpha_{1}$, or $\alpha_{2}$ (see above (51)) and $\lambda_{1} \neq 0 ; \lambda_{k}$, $k=2,3,4, \ldots$ are determined in terms of $\lambda_{0}$ and $\lambda_{1}$ and various parameters appearing in (44). For the special choice (53) of $a(x)$, we have shown that there is only one value $\mu$ equal to -1 for which the adiabatic condition (44) is satisfied, yielding a nonsingular velocity $q$. Because it does not seem possible to find the solutions of the ODE (44) explicitly, for applications to adiabatic motion this equation must be integrated numerically subject to appropriate boundary conditions. We conclude with the observation that the form of the solution (74) considered by Taylor $[2,8]$ corresponds to the special values $\alpha=$ $2 / 5$ and $g=2$ in (26)-(31).

\section{References}

1. G. C. McVitTiE, Spherically symmetric solutions of the equations of gas dynamics, Proc. R. Soc. Ser. A 220:339-355 (1953).

2. G. I. TAYLOR, The formation of a blast wave by a very intense explosion 1 . Theoretical discussion, Proc. R. Soc. Ser. A 201:159-171 (1950).

3. J. B. Keller, Spherical cylindrical and one-dimensional gas flows, Quart. Appl. Math. 14:171-184 (1956).

4. R. Courant and K. O. Friedrichs, Supersonic Flow and Shock Waves, Interscience Press, New York, 1948. 
5. K. P. Stanyukovich, Unsteady Motion of Continuous Media, Pergamon Press, New York, London, Oxford, Paris, (1960).

6. L. I. Sedov, On the integration of the equations of one-dimensional gas motion, Doklady Acad. Nauk. SSSR (N.S.) 90:735 (1953).

7. E. A. Coddington and N. Levinson, Theory of Ordinary Differential Equations, McGraw-Hill, New York, 1955.

8. J. L. TAYlor, An exact solution of the spherical blast wave problem, Phil. Mag. 46:317-320 (1955).

INDIAN INSTITUTE OF SCIENCE

Tata Institute of Fundamental Research

(Received August 31, 2004) 